\title{
The Organochlorine Pesticide Residues of Mesona Chinensis Benth by Near Infrared (NIR) Spectroscopy and Chemometrics
}

\author{
Yao Wanqing*, Zhang Shuhui, Guo Zhihui, Gu Bingming \\ School of Chemistry and Environment, Jiaying University, Meizhou, Guangdong, 514015
}

\begin{abstract}
Pesticide residue is a major problem in food safety. It is particularly important to detect pesticides in fruits and vegetables quickly and effectively. The near infrared spectroscopy (NIR) technique was explored as a technique to predict organochlorine pesticides in Mesona chinensis Benth. Fourty-three samples were sacaned powder in a Antaris II FT-NIR. Samples were split into two sets, one set used as calibration $(n=35)$ where the remaining samples $(n=8)$ were used as validation set. The hexachlorocyclohexane (BHC), dichlorodiphenyltrichloroethane (DDT) and organochlorine residues were determined by gas chromatography as the reference ratio. The prediction model of total BHC, DDT and organochlorine of mesona chinensis benth was established by partial least square (PLS) method, which were optimized by the optimal spectral band range, spectral pretreatment and regression. Moreover, the prediction ability of the model was verified by validation set. The models gave the results that RMSEC, $\mathrm{R}_{\mathrm{C}}$, RMSEP and $\mathrm{R}_{\mathrm{P}}$ of BHC, DDT and organochlorine residues respectively were 0.996, 0.9384, 0.151 and 0.9964; 1.21, $0.9889,0.409,0.9852 ; 2.21,0.9423,0.481,0.9953$. The relative errors of the predicted values are within $0.68 \% \sim 0.38 \%$ and $-0.34 \% \sim 6.88 \%$ and $-0.46 \% \sim 0.58 \%$ of the total validation set. It can be seen that the three models have good prediction effect, high precision and high stability, that is, NIR can be used as a simple, non-destructive, reliable and rapid method for the determination of organochlorine pesticide residues in mesona chinensis benth.
\end{abstract}

\section{Introduction}

Mesona chinensis Benth (Chinese Mesona), also known as xiān căo (仙草), xiānrén căo (仙人草), is an annual herb of the genus Senna in the Lamiaceae family, which is widely distributed in Guangdong, Fujian and Yunnan province in China ${ }^{[1]}$. Mesona chinensis Benth mainly contains polysaccharides, phenolic acids and flavonoids ${ }^{[2]}$. According to Traditional Chinese Medicine, Mesona chinensis Benth is cool and cold, tastes sweet and light and astringent, and has the effect of clearing heat and dampness, eliminating summer heat, quenching thirst and water, and removing heat toxicity. Mesona chinensis Benth is a natural high-grade and dual-use plant resource for medicine and food, with an extremely high nutritional value and potential health care effects ${ }^{[3]}$. However, its growth is greatly influenced by environmental factors. In order to preserve yield and increase income, growers choose pesticides that are highly efficient and low-cost, or even banned. The frequent application of pesticides resulted in exceeding standard rate of pesticide residues in Mesona chinensis Benth $^{[4]}$. Although high-residue organochlorine pesticides have been banned from production and use for up to 30 years, its satble chemical properties, long half-life, strong lipid solubility, and lack of easy degradation have resulted in serious residues in the soil, causing widespread contamination and ultimately endangering human health and trade exports [5]. According to the data from the food safety and quality supervision department, pesticide residues in vegetables and leaves in China have seriously exceeded the national standard, which has seriously harmed people's health[6]. The traditional pesticide content determination method requires a tedious and complex pretreatment process for the sample, which may damage the characteristic information of the sample. Furthermore, organochlorine pesticides commonly used in Mesona chinensis Benth mainly include benzene hexachloride (BHC) and dichlorodiphenyltrichloroethane (DDT). The analysis of these compounds by gas chromatography (GC), which requires sample preparation, specific analytical equipment and is time-consuming. For these reasons, it is extremely urgent to develop a pesticide residue measurement technology with high environmental protection, low cost and less detection time.

The Near Infrared Spectroscopy (NIR) technique comes forward as a rapid, non-destructive, large quantities, high environmental protection and low cost detection applications in technique rapidly in China in recent years ${ }^{[7]}$. In most of published workds so far, little attention has been given to the quantification of organochlorine pesticides in natural plant. Therefore, the aim of the present work was to examine the potential of NIR spectroscopy to determine the concentration of organochlorine pesticides in Mesona chinensis Benth

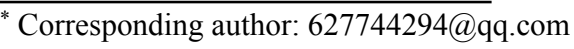


from Guang Dong.

Objective of this research is to develop and evaluate new analytical methodologies for the fast authentication of the total organochlorine residues, BHC and DDT in Chinese Mesona. Several quantitative analysis models were established by combining NIR and chemometrics methods. Via comparing and analyzing the range of different modeling characteristic bands and spectral pretreatment methods, the optimal NIR spectral models of pesticide residues are established. The above methods will attempt to identify organochlorine pesticides in samples and provide a direct, fast, simple, cheap and green alternative to chromatography for general applications, while reducing solvent consumption and waste generation.

\section{Materials and methods}

\subsection{Experimental materials}

Forty-three Mesona chinensis Benth samples were dyred, removing impurities and root, leaving stems and leaves. The samples were split into two sets, 35 samples were used in calibration, where the remaining 8 samples used as external validation set. All the samples were grown at Guangdong and harvested during summer and autumn in different years (2018-2019).

\subsection{Experimental reagents}

Petroleum ether $\left(60-90{ }^{\circ} \mathrm{C}\right)$, sulfuric acid, sodium chloride, anhydrous sodium sulfate, dichloromethane, acetone, all were analytically pure.

\subsection{Experimental apparatus}

Antaris II Fourier transform infrared spectrometer (FT-NIR), Thermo Fisher Scientific (China) Co., Ltd; GC-2010 gas chromatograph, Shimadzu Enterprise Management (China) Co., Ltd; low-speed benchtop centrifuge, Jiangsu Jinyi Instrument Technology Co., Ltd; $\mathrm{CNC}$ ultrasonic cleaner, Kunshan Ultrasonic Instrument Co., Ltd; rotary evaporator, Shanghai Yarong Biochemical Equipment and Instrument Factory; ultrapure water machine, Sichuan Excellence Water Treatment Equipment Co.

\subsection{Experimental methods}

\subsubsection{Gas chromatography analysis}

(1) Gas chromatographic conditions

Chromatographic conditions: column: The gas chromatograph equipped with a splitl/splitless injector, an electron capture detector (63 Ni-ECD) and an quartz capillary column (HP-7,30 $\mathrm{m} \times 0.32 \mathrm{~mm}, 0.25 \mu \mathrm{m})$. The temperature of the injector and detector is set to $230{ }^{\circ} \mathrm{C}$ and $300^{\circ} \mathrm{C}$. The oven temperature was initially held at $100{ }^{\circ} \mathrm{C}$ for 5 minutes, then programmed to rise to $220^{\circ} \mathrm{C}$ at $10{ }^{\circ} \mathrm{C}$ per minute and then programmed to rise to
$250{ }^{\circ} \mathrm{C}$ at $8{ }^{\circ} \mathrm{C}$ per minute. Finally maintained at $250{ }^{\circ} \mathrm{C}$ for 10 minutes. Carrier gas nitrogen (purity $>99.999 \%$ ) has an initial flow rate of $1 \mathrm{~mL}$ per minute and the makeup flow rate was $58.5 \mathrm{~m}$ Lper minute. The analyses were performed by injecting $1 \mu \mathrm{L}$ of sample in splitless mode $(15 \mathrm{~mL} / \mathrm{min})$ under the constant pressure mode.

Standard curve plotting: Petroleum ether $\left(60-90{ }^{\circ} \mathrm{C}\right)$ was used as the solvent to configure the standard solutions of nine organochlorine mixed pesticide controls at a concentration of $0.01 \mathrm{~mL} / \mathrm{mL}$. According to library searching or structure elucidation, the standard working curve was drawn by the normalization method of peak area.

Determination of samples: Petroleum ether $\left(60-90^{\circ} \mathrm{C}\right)$ was used as the solvent to configure the tested samples at $0.2 \mathrm{~mL} / \mathrm{mL}$ and diluted 5 times. The gas chromatographic conditions were the same as those used for the standard curve, and 35 samples of Mesona chinensis Benth were determined. According to the peak area of each indicator measured, the conversion was calculated on the standard working curve to obtain the residues of nine organochlorine pesticides in the samples.

(2) Preparation of mixed solution control

$0.5 \mathrm{~mL}$ of total hexhexane (BHC: $\alpha$-BHC, $\beta$-BHC, $\gamma$-BHC, $\Delta$-BHC), DDT (DDT: P, P'-DDE, P, P '-DDD, O, $\mathrm{P}$ '-DDT, P, P '-DDT) and pentachloronitrobenzene (PCNB) mixed pesticide reference were measured and placed in a $50 \mathrm{~mL}$ volumetric flask Dilute with petroleum ether $\left(60 \sim 90^{\circ} \mathrm{C}\right)$ to the marking line, shake well, that is, the mixed pesticide control solution.

(3) Preparation of the test solution

Referring to the 2015 version of the Chinese Pharmacopoeia method ${ }^{[8]}$, take test article of Mesona chinensis Benth, crushed into powder (sieve No. 3). Precise weighing $2 \mathrm{~g}$, put it in a $100 \mathrm{~mL}$ stopper conical flask, add $20 \mathrm{~mL}$ water and soak overnight, Precisitely add $40 \mathrm{~mL}$ acetone for ultrasonic treatment for 30 minutes, let it cool down, and then add about $6 \mathrm{~g}$ sodium chloride Afterwards, precisely add $30 \mathrm{~mL}$ dichloromethane, ultrasound for $15 \mathrm{~min}$, and let stand to stratify The organic phase was quickly transferred into a $100 \mathrm{~mL}$ stopper conical flask containing an appropriate amount of anhydrous sodium sulfate and placed for 4 hours. Measure $35 \mathrm{~mL}$ precisely, concentrate under reduced pressure on a water bath at $40^{\circ} \mathrm{C}$ until nearly dry. Add a small amount of petroleum ether $\left(60-90^{\circ} \mathrm{C}\right)$ as before repeatedly until dichloromethane and acetone are removed. Dissolve with petroleum ether $\left(60-90^{\circ} \mathrm{C}\right)$ and transfer to a $10 \mathrm{~mL}$ stoppered graduated centrifuge tube. Add petroleum ether and dilute precisely to $5 \mathrm{~mL}$, carefully add sulfuric acid $1 \mathrm{~mL}$, shake for $1 \mathrm{~min}$, and centrifuge $(3000 \mathrm{rpm})$ for $10 \mathrm{~min}$. The supernatant was precisely measured by $2 \mathrm{~mL}$, connected to a rotary evaporator, and the solution was concentrated to an appropriate amount at $40^{\circ} \mathrm{C}$ and precisely diluted to 1 $\mathrm{mL}$. That is, all. 


\subsubsection{Establishment of NIR quantitative analysis model}

Near infrared (NIR) spectra were recorded on a Antaris II Fourier transform infrared spectrometer equipped with an integrating sphere diffuse reflectance module, from 10000 to $4000 \mathrm{~cm}^{-1}$. For each sample, 64 scans were made with a spectral resolution of $8 \mathrm{~cm}^{-1}$ and 4 times gain, then averaged. Samples were temperature equilibrated at $24^{\circ} \mathrm{C}$ (approximately $3 \mathrm{~min}$ ) in the instrument before scanning. Ominic software (Thermo Electron Corporation) was used for spectrometer configuration, control, and data acquisition. Three parallel samples were taken for each sample (reloading). Three NIR spectra were collected for each sample, 3 for each sample, for a total of 129 spectra. 105 spectra were used as the calibration set and 24 spectra were used as the validation set.

The quantitative models of organochlorine residue in Mesona chinensis Benth were established by TQ spectral analysis software (Thermo Electron Corporation). The precision measurement standards of the optimal quantitative analysis models mainly include the calibration correlation coefficient $\mathrm{R}_{\mathrm{C}}$, the prediction correlation coefficient $\left(\mathrm{R}_{\mathrm{P}}\right)$, the cross-validation correlation coefficient $\left(\mathrm{R}_{\mathrm{CV}}\right)$ and the calibration mean square deviation (RMSEC), the prediction mean square deviation (RMSEP) and the cross-validation mean square deviation (RMSECV) of the model, the performance index of the model, and the prediction effect of the model.

\subsubsection{Application of the quantitative model}

Samples No. 4 , No. 17 , No. 18 , No. 20 , No. 25 , No. 26 , No. 29 and No. 33 (randomly selected), which were not involved in the modeling, were used as the validation sample set and imported into the chemometric model built in 4.2 for external detection. The residues predicted by the model were compared with the actual residues determined by GC to test and evaluate the prediction performance of the built model.

\section{Results and Discussion}

\subsection{Gas chromatography analysis}

On the basis of the current Green Industry Standard for Import and Export of Medicinal Plants and Preparations and 2015 edition of Chinese Pharmacopoeia limit indexes, the residues of total $\mathrm{BHC}$, total DDT and PCNB shall not exceed $100 \mathrm{mg} / \mathrm{g}^{[8-9]}$.

The residues of total BHC ( $\alpha$-BHC, $\beta$-BHC, $\gamma$-BHC, $\delta$-BHC), total DDT (p,p'-DDE, p,p'-DDD, o,p'-DDT, $\mathrm{p}, \mathrm{p}^{\prime}$-DDT) and PCNB of nine organochlorine pesticides in 35 samples were determined and calculated by gas chromatography combined with reference methodology. The results in table1 showed that the total residues of organochlorine pesticides in the samples were within the standard range, which the total amount is ranged from 0 to $34.81 \mathrm{mg} / \mathrm{g}$. Besides, for the studied compounds, the residues of total BHC, total DDT and PCNB were ranged from $1.28 \mathrm{mg} / \mathrm{g}$ to $22.93 \mathrm{mg} / \mathrm{g}$, from $0.14 \mathrm{mg} / \mathrm{g}$ to $24.74 \mathrm{mg} / \mathrm{g}$ and from $0.50 \mathrm{mg} / \mathrm{g}$ to $2.15 \mathrm{mg} / \mathrm{g}$, respectively. The residual amount of total $\mathrm{BHC}$ and total DDT in samples based on GC analysis were high, while the residual amount of PCNB residues was low In this reason, that is why PCNB residues were out of discussion here.

\subsection{Near-infrared spectra}

Fig. 1 shows the NIR spectra of 43 Chinese Mesona samples without any data pre-treatment in the range between 10000 to $4000 \mathrm{~cm}^{-1}$. The absorption band at $4660 \mathrm{~cm}^{-1}$ corresponds to the $\mathrm{C}-\mathrm{C}$ and $\mathrm{C}-\mathrm{H}$ stretching vibration, while strong bands at $5270 \mathrm{~cm}^{-1}$ corresponds to the $\mathrm{C}-\mathrm{Cl}$ absorption of organochlorine compound. Absorption bands at $5790 \mathrm{~cm}^{-1}$ are related with first order octavo absorption due to $\mathrm{C}-\mathrm{H}$ stretching vibrations of methyl groups attached to an aromatic, $6950 \mathrm{~cm}^{-1}$ related with synchronous frequency absorption of cyclohexane $\mathrm{C}-\mathrm{H}$ stretching vibration ${ }^{[10]}$. The measurement results are affected by external factors such as the density of the samples in the sample cup, the shape and size of the sample during the collection of the spectra. Given the results, this comes as no surprise that reproducibility of the spectra was mediocre.

Table 1 The Organochlorine Pesticide Residues of Mesona Chinensis Benth

\begin{tabular}{|c|c|c|c|c|c|c|c|c|c|}
\hline \multirow[b]{2}{*}{ sample } & \multicolumn{4}{|c|}{ Amount of residue $\mu \mathrm{g} / \mathrm{kg}$} & \multirow[b]{2}{*}{ sample } & \multicolumn{4}{|c|}{ Amount of residue $\mu \mathrm{g} / \mathrm{kg}$} \\
\hline & $\begin{array}{l}\text { Total } \\
\text { BHC }\end{array}$ & $\begin{array}{l}\text { Total } \\
\text { DDT }\end{array}$ & PCNB & $\begin{array}{c}\text { total } \\
\text { organochlorine } \\
\text { pesticides }\end{array}$ & & $\begin{array}{l}\text { Total } \\
\text { BHC }\end{array}$ & $\begin{array}{l}\text { Total } \\
\text { DDT }\end{array}$ & PCNB & $\begin{array}{c}\text { total } \\
\text { organochlorine } \\
\text { pesticides }\end{array}$ \\
\hline 1 & 5.83 & 1.14 & - & 6.97 & 19 & 5.08 & 0.14 & - & 5.22 \\
\hline 2 & 3.39 & 11.82 & - & 15.21 & 20 & 7.59 & 1.67 & - & 9.26 \\
\hline 3 & 1.28 & - & - & 1.28 & 21 & 2.26 & 0.39 & - & 2.65 \\
\hline 4 & 8.77 & 1.46 & - & 10.23 & 22 & 9.57 & 24.74 & 0.50 & 34.81 \\
\hline 5 & 6.45 & - & - & 6.45 & 23 & 22.93 & 1.45 & - & 24.38 \\
\hline 6 & 1.91 & - & 0.95 & 2.86 & 24 & 17.98 & 14.86 & - & 32.84 \\
\hline 7 & 4.03 & - & 2.26 & 6.29 & 25 & 13.75 & 4.75 & - & 18.50 \\
\hline 8 & 7.19 & 0.41 & - & 7.60 & 26 & 9.23 & 1.30 & - & 10.53 \\
\hline 9 & - & 0.41 & - & 0.41 & 27 & - & 8.96 & - & 8.96 \\
\hline 10 & 3.02 & 0.30 & - & 3.32 & 28 & - & 6.80 & - & 6.80 \\
\hline
\end{tabular}




\begin{tabular}{|c|c|c|c|c|c|c|c|c|c|}
\hline 11 & 3.40 & 1.04 & - & 4.44 & 29 & 2.24 & 3.99 & - & 6.23 \\
\hline 12 & - & 0.73 & - & 0.73 & 30 & 1.48 & 5.50 & - & 6.98 \\
\hline 13 & 285.9 & 0.73 & - & 29.32 & 31 & 4.92 & 7.22 & - & 12.14 \\
\hline 14 & - & - & - & - & 32 & 7.09 & 7.05 & 0.89 & 15.03 \\
\hline 15 & 1.86 & - & - & 1.86 & 33 & 9.62 & 3.38 & - & 13.00 \\
\hline 16 & - & 4.16 & - & 4.16 & 34 & 16.09 & 0.45 & 2.15 & 18.69 \\
\hline 17 & 1.91 & 29.21 & - & 31.12 & 35 & 8.97 & 1.87 & 1.11 & 11.95 \\
\hline 18 & 4.88 & 0.52 & - & 5.40 & & & & & \\
\hline
\end{tabular}

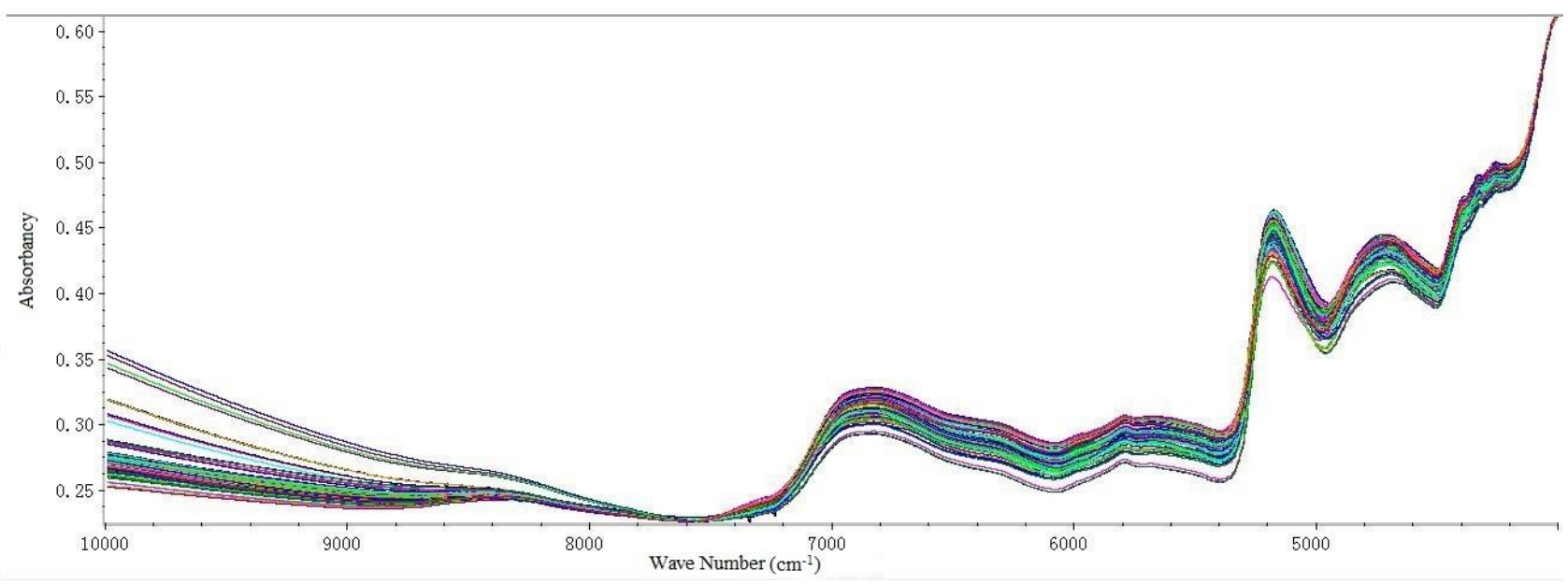

Fig. 1 Near infrared spectra of all samples

\subsection{Selection of the best model for Chinese Mesona authentication}

Several chemometric modes were assayed employing different spectral regions and data pre-treatments, including multiplicative scatter correction (MSC), Savitaky-Golay filter (SG) first (FD) and second (SD) derivative, and Norris-derivative filter (ND), standard normal variation (SNV) mean centering (MC) and autoscale, together with combinations between them.

The optimal modeling characteristic bands were selected, combined with the optimal spectral pretreatment method, and the partial least square method (PLS) was adopted to establish the quantitative analysis models of total organic chlorine (Figure 2), DDT (Figure
3 ) and BHC (Figure 4).

The relationship between the model predicted values of total organochlorine residues, total DDT and BHC and the measured values of GC is shown in Figure 2, Figure 3 and Figure 4, respectively. Table 2 shows the descriptive statistics for the organochlorine pesticide residue parameters used for either the calibration or the validation set. The best coefficient of determination in calibration $\left(\mathrm{R}_{\mathrm{c}}\right)$ and the lowest standard error in cross validation (SECV.) were obtained after applying the Savitzky Golay (SG) and second (SD) derivative and using the optimal band range by PLS method. Regarding data shown, for total organic chlorine the $R_{c}$ was 0.9423 (RMSECV: 0.481); for total DDT the $\mathrm{R}_{\mathrm{c}}$ was 0.9887 (RMSECV: 0.426); for total $\mathrm{BHC}$ the $\mathrm{R}_{\mathrm{c}}$ was 0.9384(RMSECV: 0.151).

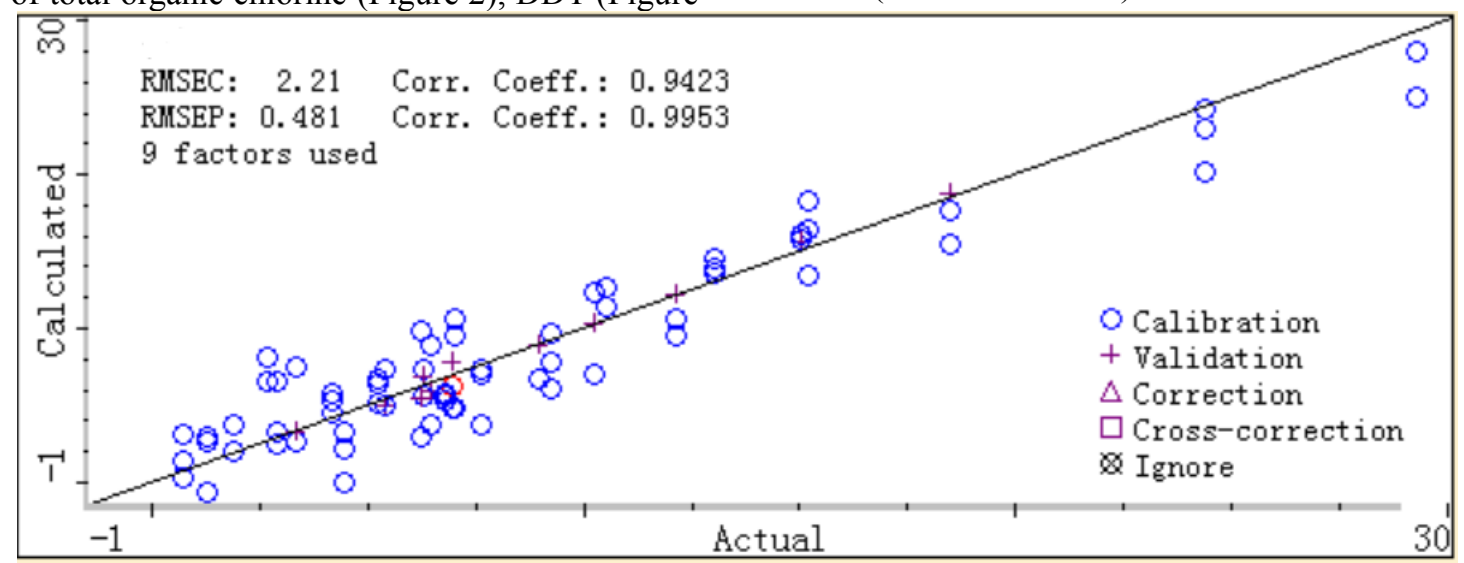

Figure 2 NIR model of total organochlorine residues 


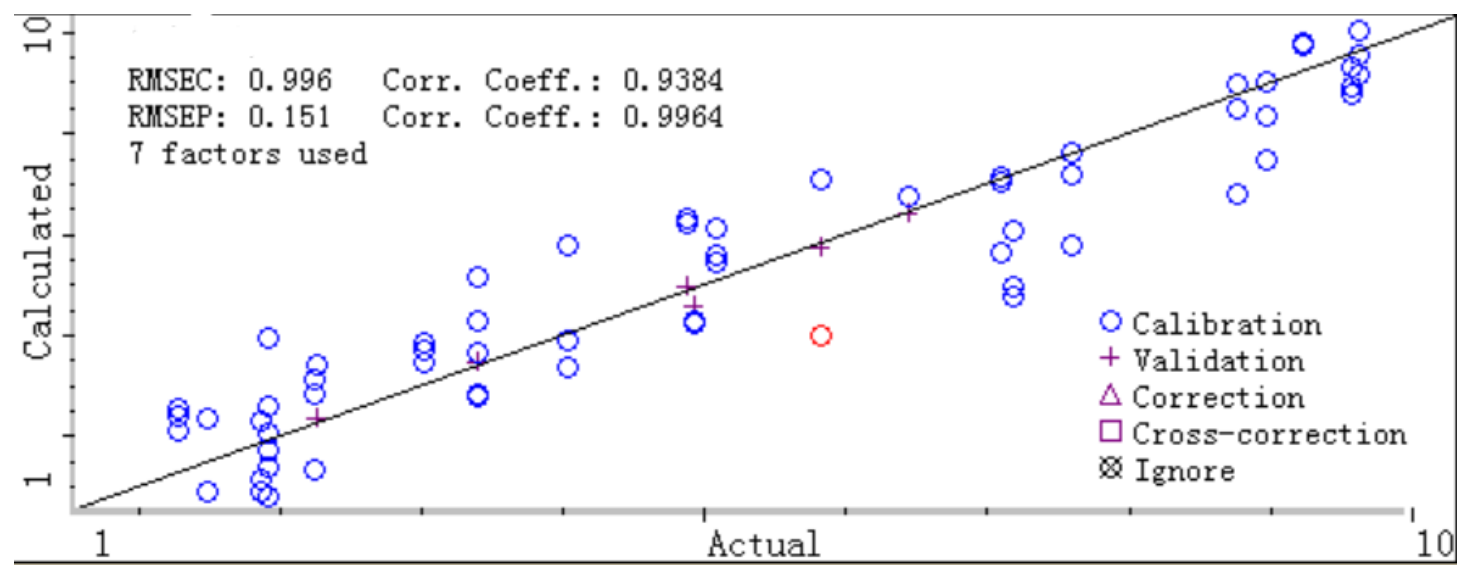

Figure 3 NIR model of total BHC residues

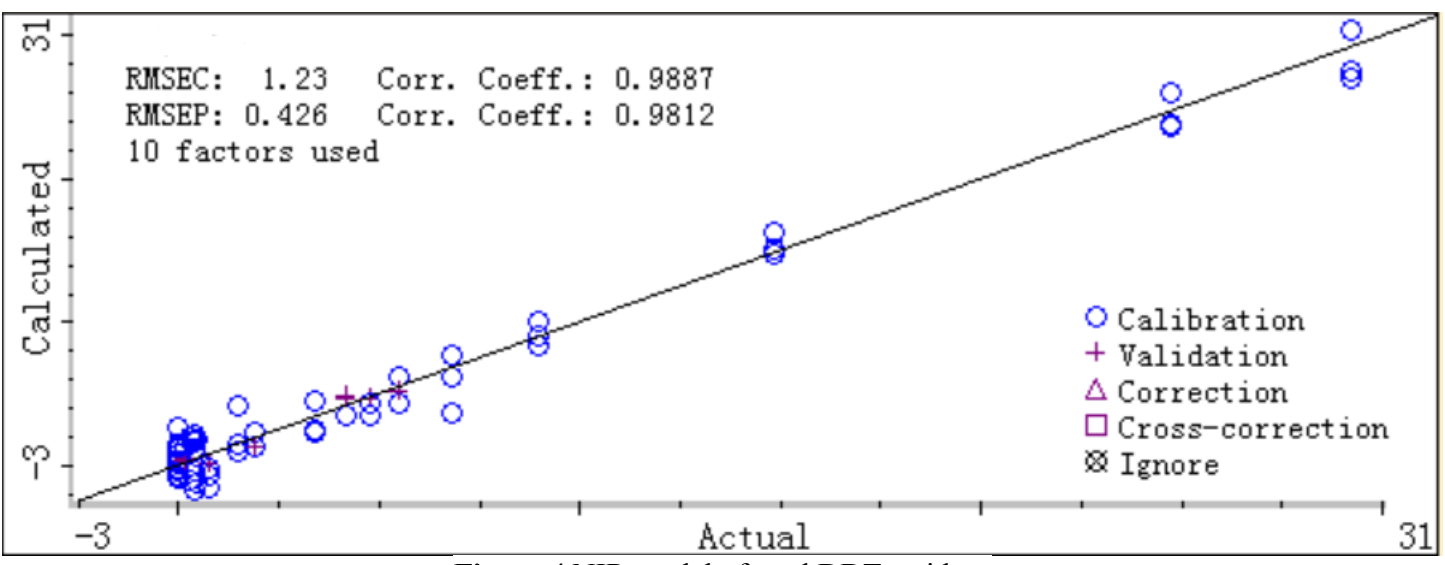

Figure 4 NIR model of total DDT residues

Table 2 Calibration and cross validation statistics for organochlorine pesticide residues on chinese mesona herb samples

\begin{tabular}{|c|c|c|c|c|c|c|c|c|c|}
\hline Parameter & $\begin{array}{l}\text { Preprocessing } \\
\text { method }\end{array}$ & Modeling band $\left(\mathrm{cm}^{-1}\right)$ & RMSEC & $\mathrm{R}_{\mathrm{C}}$ & RMSEP & $\mathrm{R}_{\mathrm{P}}$ & RMSECV & $\mathrm{R}_{\mathrm{CV}}$ & $\begin{array}{c}\text { Perfor } \\
\text { mance } \\
\text { index }\end{array}$ \\
\hline Total BHC & $\begin{array}{l}\text { Second order } \\
\text { derivative } \\
\text { (SG filtered } \\
\text { dry) }\end{array}$ & $\begin{array}{l}5289.57-4735.18 \\
5635.13-5329.63 \\
6356.23-5823.54 \\
7467.70-6984.22\end{array}$ & 0.996 & 0.9384 & 0.151 & 0.9964 & 2.58 & 0.5296 & 98.0 \\
\hline Total DDT & $\begin{array}{c}\text { Second order } \\
\text { derivative } \\
\text { (SG filtered } \\
\text { dry) }\end{array}$ & $5199.95-4086.02$ & 1.23 & 0.9887 & 0.426 & 0.9852 & 4.64 & 0.8292 & 94.9 \\
\hline $\begin{array}{l}\text { Total } \\
\text { organochlo } \\
\text { rines }\end{array}$ & $\begin{array}{l}\text { Second order } \\
\text { derivative } \\
\text { (SG filtered } \\
\text { dry) }\end{array}$ & $\begin{array}{l}4961.73-4449.88 \\
5401.71-5262.43 \\
6095.53-5879.11\end{array}$ & 2.21 & 0.9423 & 0.481 & 0.9953 & 5.43 & 0.6645 & 94.9 \\
\hline
\end{tabular}

\subsection{Prediction capability of NIR models}

To evaluate the predictive capability of the PLS models, a set of validation samples $(n=8)$ were introduced into the developed PLS models. According to the results obtained by GC, table 3 summarizes the parameter range of variation for reference values, predicted values and predicted values. As it can be seen, compare to relative deviation for total DDT, better prediction for DHC and total organochlorines in prediction, with a precision range spanning values between $-0.68 \%$ and $0.38 \%,-0.46$ and $0.58 \%$, respectively. In short, it can be concluded that the developed PLS models provides a good prediction capability for total DDT, DHC and total organochlorines. 
Table 3 Prediction results of NIRS models

\begin{tabular}{ccccc}
\hline Parameter & $\begin{array}{c}\text { Range of variation of } \\
\text { reference values } \\
(\mathrm{mg} / \mathrm{g})\end{array}$ & $\begin{array}{c}\text { Range of variation } \\
\text { of predicted values } \\
(\mathrm{mg} / \mathrm{g})\end{array}$ & $\begin{array}{c}\text { Range of } \\
\text { deviation values } \\
(\mathrm{mg} / \mathrm{g})\end{array}$ & $\begin{array}{c}\text { Relative } \\
\text { deviation }(\%)\end{array}$ \\
\hline Total DHC & $1.91 \sim 13.75$ & $1.36 \sim 10.06$ & $-9.82 \sim 1.46$ & $-0.68 \sim 0.38$ \\
\hline Total DDT & $1.30 \sim 29.21$ & $2.10 \sim 30.63$ & $-2.42 \sim 5.47$ & $-0.34 \sim 6.88$ \\
\hline $\begin{array}{c}\text { Total } \\
\text { organochlorines }\end{array}$ & $5.40 \sim 31.12$ & $2.94 \sim 20.95$ & $-14.43 \sim 3.61$ & $-0.46 \sim 0.58$ \\
\hline
\end{tabular}

\section{Conclusions}

The development and evaluation of chemometric Near Infrared spectroscopy based models has evidenced the possibility of prediction of organochlorine pesticides in Mesona chinensis Benth through the use of a PLS model established from well characterized samples by a reference methodology and the appropriate chemometric treatment. As shown and from the Green Analytical point of view, the use of infrared spectroscopy together with chemometric treatment of data reduces the use of reagents and waste generation when compared to the reference methodology, thus these methods can be considered as environmentally friendly alternative methodologies for determination of the residues of total DHC, total DDT and total organochlorines in mesona chinensis benth sample.

\section{Acknowledgement}

Foundation item: Project of science and Technology Assisting Agriculture of rural science and Technology Commissioner in Guangdong Province "The characteristics of agricultural products storage and preservation and downstream products of mechanical processing and promotion in Wenshui village", Science and Technology Program of Guangdong Province (2020B121201013): Guangdong Provincial Key Laboratory of Conservation and Precision Utilization of Characteristic Agricultural Resources in Mountainous Areas, Key research project of JiaYing University (2019KJZ02).

\section{References}

1. Editorial Committee of Flora of China, Chinese Academy of Sciences. Flora of China [M]. Beijing: Science Press, 1997:1849.

2. Liu Sulian. Preliminary Study on the Chemical Composition of Ligustrum Lucidum[J]. Chinese Medicinal Materials, 1995(5):247-248.

3. National Compendium of Chinese Herbs Preparation Group. National Compilation of Chinese Herbal Medicines [M]. The second volume. Beijing: People's Health Publishing House, 1978:474.

4. Wang Lili, Xia Huilong. Characteristics of Pesticide Residues in Chinese Herbal Medicines in China[J]. Chinese Herbal Medicine,2017,38(3):471.

5. Yang Yinhui, Dou Xiaowen, Kong Weijun, et al.
Current Status and Pollution Analysis of Pesticide Registration in Chinese Herbal Medicines in China[J]. Chinese Journal of Traditional Chinese Medicine,2013,38(24):4238.

6. Liu Kai, Analysis of Spectral Characteristics of Organophosphorus Pesticide Residues in Vegetables[J], Bulletin of science and technology, 2017, 33 (8): 238-242.

7. Genisheva, Z, Quintelas, C, Mesquita, D.P,,et al. New PLS analysis approach to wine volatile compounds characterization by near infrared spectroscopy(NIR) [J]. Food Chemistry, (246) 2018: $172-178$.

8. National Pharmacopoeia Committee. Pharmacopoeia of the People's Republic of China (four parts) [M]. Beijing: China Pharmaceutical Science and Technology Press, 2015:206.

9. Ministry of Foreign Trade and Economic Cooperation of the People's Republic of China. Green Industry Standards for the Import and Export of Medicinal Plants and Preparations [S].2001,121.

10. Jere Workman, Lois Vinyi. A Practical Guide to Near-infrared Spectroscopy [M]; Chu, S. L., Xu, Y. P., and Tian, G. Y. Translation. Beijing: Chemical Industry Press,2009. 